\title{
Movement Disorders in patients with alternating hemiplegia
}

"soft" and "stiff" at the same time

Eleni Panagiotakaki ${ }^{1^{*}}$, Diane Doummar ${ }^{\star}$, Erika Nogue ${ }^{3}$, Nicolas Nagot $^{3}$, Gaetan Lesca ${ }^{4}$, Florence Riant $^{5}$, Sophie Nicole ${ }^{6}$, Charlene Delaygue ${ }^{7}$, Marie Anne Barthez ${ }^{8}$, Marie Cécile Nassogne ${ }^{9}$, Anne Dusser ${ }^{10}$, Louis Vallée ${ }^{11}$, Thierry Billette ${ }^{2}$, Marie Bourgeois ${ }^{12}$, Christine $\operatorname{loos}^{13}$, Cyril Gitiaux ${ }^{14}$, Cécile Laroche ${ }^{15}$, Mathieu Milh ${ }^{16}$, Vincent Des Portes ${ }^{17}$, Alexis Arzimanoglou ${ }^{1}$, Agathe Roubertie ${ }^{7,18}$, the AHC-Movement Disorder Study Group

1. Department of Paediatric Clinical Epileptology, sleep disorders and Functional Neurology, University Hospitals of Lyon; Member of the ERN EpiCARE, Lyon, France

2. Service de Neurologie Pédiatrique, Hôpital Trousseau, APHP, Paris, France

3. Centre d'Investigation Clinique, CHU Montpellier, Montpellier, France

4. Hospices Civils de Lyon, Department of Medical Genetics, Centre de Biologie Est, Lyon University Hospital, Member of the ERN EpiCARE, Lyon, France

5. Laboratoire de Génétique, Groupe hospitalier Lariboisière-Fernand Widal AP-HP, Paris, France

6. IGF, Univ. Montpellier, CNRS, INSERM, Montpellier, France

7. Département de Neuropédiatrie, CHU Gui de Chauliac, Montpellier, France

8. Service de Neuropédiatrie et Handicaps, Hôpital Gatien de Clocheville, CHU Tours, France.

9. Pediatric Neurology Unit, Cliniques Universitaires Saint-Luc, UCLouvain, Brussels, Belgium

10. Service de Neuropédiatrie, CHU de Bicêtre, Kremlin-Bicêtre, France

11. Service de Neuropédiatrie, CHU Lille, Lille, France

12. Service de Neurochirurgie pédiatrique, Hôpital Necker-Enfants Malades, APHP, Paris, France

13. Service de Neurologie Pédiatrique, Hôpiutal Raymond Poincarré, AP-HP,Garches, France

14. Service de neurophysiologie, Hôpital Necker, AP-HP, Paris, France

15. Département de Pédiatrie, CHU Limoges, Limoges, France

16. Service de Neurologie Pédiatrique, CHU Timone Enfants, Marseille, France

17. Centre de référence " Déficiences Intellectuelles de causes rares », Hôpital Femme Mère Enfant, Hospices Civils de Lyon, F-69677 Bron, France, Université de Lyon, F-69008 Lyon, France

18. INSERM U 1051, Institut des Neurosciences de Montpellier, Montpellier, France

\section{Corresponding author:}


Professor Agathe Roubertie, Département de Neuropédiatrie, CHU Gui de Chauliac, 80 Avenue Fliche, 34295 Montpellier, France

Tel: 33467330182 ; fax: 33467330183

a-roubertie@chu-montpellier.fr

https://orcid.org/0000-0002-8180-4857

\section{Supplemental Data}

Video case reports: video1, video2 and video3; legends to the videos.

\section{Statistical analysis}

Statistical analysis were performed by Erika Nogue and Professor Nicolas Nagot

Centre d'Investigation Clinique, CHU Montpellier, Montpellier, France

\section{Counts}

word count for the paper : 2625

word count for abstract : 238

character count for the title (including spaces and punctuation) : 90

number of references: 25

number of tables : 2

number of figures : 2

\section{Study funding : none}

Search terms : dystonia (162) ; chorea (170) ; developmental disorders (228) ;mental retardation (229); ;ion channel gene defects $(97)$

\section{Disclosure Statement}

Dr. Eleni Panagiotakaki, Diane Doummar, Nicolas Nagot, Gaetan Lesca Florence Riant, Sophie Nicole, Alexis Arzimanoglou, Marie Anne Barthez, Marie-Cécile Nassogne, Anne Dusser, Louis Vallée, Thierry Billette, Marie Bourgeois, Christine loos, Cyril Gitiaux, Cécile Laroche, Mathieu Milh, Vincent Desportes, Erika Nogue, Charlene Delaygue and Pr Agathe Roubertie, reports no disclosures 


\section{Abstract}

AIM: To assess non-paroxysmal movement disorders in ATP1A3 mutation-positive patients with alternating hemiplegia of childhood $(\mathrm{AHC})$.

METHODS: Twenty-eight patients underwent neurological examination with particular focus on movement phenomenology by a specialist in movement disorders. Video recordings were reviewed by another movement disorders specialist and data were correlated to patients' characteristics.

RESULTS: Ten patients were diagnosed with chorea, 16 with dystonia (nonparoxysmal), 4 with myoclonus, and 2 with ataxia. Nine patients had more than one movement disorder and 8 patients had none. The degree of movement disorder was moderate to severe in 12/28 patients. At inclusion, dystonic patients $(n=16)$ were older $(p=0.007)$ than nondystonic patients. Moreover, patients $(n=18)$ with dystonia or chorea, or both, had earlier disease onset $(p=0.042)$ and more severe neurological impairment $(p=0.012)$, but this did not correlate with genotype. All patients presented with hypotonia, which was characterized as moderate or severe in $16 / 28$. Patients with dystonia or chorea $(n=18)$ had more pronounced hypotonia $(p=0.011)$. Bradykinesia $(n=16)$ was associated with an early age at assessment $(p<0.01)$. Significant dysarthria was diagnosed in $11 / 25$ cases. A history of acute neurological deterioration and further regression of motor function, typically after a stressful event, was reported in 7 patients.

INTERPRETATION: Despite the relatively limited number of patients and the cross-sectional nature of the study, this detailed categorisation of movement disorders in patients with AHC offers valuable insight into their precise characterization. Further longitudinal studies on this topic are needed. 


\section{Introduction}

Alternating hemiplegia of childhood $(\mathrm{AHC})$ is a rare genetic condition, primarily caused by de novo mutations in the ATP1A3 gene (AHC2, MIM 182350). AHC is characterized by a complex phenotype that combines paroxysmal epileptic and/or non-epileptic episodes, developmental abnormalities, and permanent neurological impairment with hypotonia, gross and fine motor

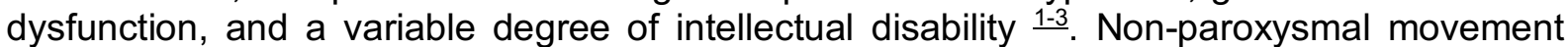
disorders (NPMDs) also represent prominent features of the AHC phenotype, especially chorea and dystonia, which were reported in $75 \%$ of a large European cohort 4 ..

To date, the majority of studies concerning AHC were dedicated to hemiplegic attacks and other paroxysmal phenomena. More recently, published studies have attempted to better characterize the non-paroxysmal phenomena in patients with $\mathrm{AHC}$ in terms of motor function $\underline{5}$ and cognitive and behavioural profiles $\underline{6}$ but there is a lack of data focusing on the nature and characteristics of NPMDs. The aim of this study was to asses NPMDs in a group of patients with AHC, defined by Aicardi's criteria and harbouring a pathogenic ATP1A3 mutation, with special focus on (i) the impact of movement disorders, especially dystonia and/or chorea, on global neurological impairment; (ii) the association between movement disorders and other clinical symptoms; (iii) the hypothesized correlation between genotype and occurrence of movement disorders

\section{Patients and Methods}

\section{Standard Protocol Approvals, Registrations, and Patient Consents}

This study is based on the database of the nEUroped project, an EU funded project in which clinical data were collected from AHC patients from different European countries and which was performed as part of the research activities of the International Consortium for Research and Care for ATP1A3 diseases (http://www.iahcrc.net). Our study was carried out in accordance with the Declaration of Helsinki and approved by Ethical Committees (Comité consultatif sur le traitement de l'information en matière de recherche dans le domaine de la santé - Advisory Committee on the Treatment of Information in Health Research, number 11.463bis). Our study was carried out in accordance with the Declaration of Helsinki and approved by Ethical Committees. Written informed consent was obtained from the patients' parents or legal representatives for clinical data collection, genetic studies, and publication of the results. Video authorization has been obtained for disclosure (consent-to-disclose).

\section{Clinical assessment}

French patients with AHC, fulfilling Aicardi's criteria ${ }^{7}$, and harbouring one ATP1A3 pathogenic mutation (previously enrolled in the nEUroped project together with some newly diagnosed cases), were included in this cross-sectional study. Data collection was performed by three clinicians (EP, DD, and AR). Information on past medical history was collected based on medical files, parental interviews, and the nEUroped database; information regarding clinical status at inclusion was collected using a standardized questionnaire (to determine the occurrence of paroxysmal events including epileptic seizures, current treatment, and autonomy in daily-living activities). Neurological examination was performed by an expert in AHC (EP) and a specialist in movement disorders (AR), with particular focus on the phenomenology of movement disorders assessed according to an established classification $\stackrel{8}{*}$. The findings were reviewed by another specialist in movement disorders (DD), based on video recordings obtained with written informed consent. The severity of global neurological impairment was assessed according to the 'non-paroxysmal disability index' (DI) described by Panagiotakaki et al. ${ }^{4}$ (table 1 ). Speech intelligibility was assessed using a simple 4-point 
scoring system (table 1). Moreover, we decided to assign patients to 4 distinct groups reflecting their degree of hypotonia, as this would be appreciated by clinical examination: Normal tonus ; Mild hypotonia if the patient had an abnormal tonus in passive mobilization of the limbs; Moderate hypotonia, if hypotonia seemed to influence gait, making it difficult; and Severe hypotonia when perceived hypotonia during clinical examination would be so pronounced that patients would be unable to sit or hold their head.

\section{Statistical analysis}

Patients' characteristics are presented using median and interquartile range (IQR 25-75 $^{\text {[or mean }}$ $\pm S D]$ ) for continuous variables, and frequencies and proportions for categorical variables.

Groups (defined by severity of AHC, abnormal movement, hypotonia, or ATP1A3 mutation) were compared using the Student or Wilcoxon rank test for continuous variables and Chisquare or Fisher test for categorical variables. The statistical bilateral significance threshold was set at 5\%. Statistical analyses were performed using SAS version 9.3 (SAS Institute, Cary, North Carolina).

Data availability

Anonymized data will be shared by the corresponding author after request from any qualified investigators.

\section{Results}

\section{Population: general characteristics}

Twenty-eight patients (15 male and 13 female) were enrolled. The median age at inclusion was 11 years $\left(\mathrm{IQR}_{25-75}: 4.4-18.8\right.$; range: $\left.1.8-38.6\right)$. Clinical and genetic characteristics of patients are summarized in table 2 . The median disability index (DI) was 8/16 (IQR $25-75: 5$ - 10; range: 2-15). Distribution of the patients according to the value of the subscore is presented in table 2. Hypotonia was assessed in all patients, with a clinical appreciation as mild in 12 patients, moderate in 11 patients, and severe in 5 patients. Significant dysarthria or impaired speech intelligibility was diagnosed in 11/25 cases (information not collected for three patients). Medical history revealed that 7 patients exhibited acute neurological deterioration and further regression of motor function (typically after a stressful event). All those patients lost motor achievements and they did not regain prior level of function.

At enrollment, a median of 2 different drugs per patient was used, with a maximum of 5 for 1 patient. Twenty-one patients were on flunarizine, 7 benzodiazepines, 6 sodium valproate, 4 topiramate, and 2 levetiracetam; other antiepileptic drugs included carbamazepine, phenytoin, phenobarbital, lamotrigine, vigabatrin, zonisamide, and pregabalin. One patient benefited from trihexyphenidyl. Three patients received no treatment.

\section{Nonparoxysmal movement disorders}

Three illustrative cases with video recordings of nonparoxysmal movement disorders are available in Supplementary Material (videos 1-3).

Clinical examination led to the diagnosis of various forms of hyperkinetic movement disorders (figure 2): chorea ( $n=10$ patients), dystonia (nonparoxysmal) $(n=16)$, myoclonus $(n=4)$, and ataxia $(n=2)$. A combination of more than 1 movement disorder was established in 9 patients, whereas 8 patients exhibited no movement disorders according to medical files and at assessment. Bradykinesia was noticed in 16 patients, including 10 who also exhibited dystonia 
(5 isolated, 4 with choreo-dystonia, and 1 with dystonia and ataxia), 2 with myoclonus, and 4 without hyperkinetic movement disorder. Movement disorders were assessed as moderate to severe in 12/28 patients. A rostrocaudal gradient was assessed in 21 patients and was present in $15(71.4 \%)$.

\section{Comparative analysis of the disease disability index, movement disorders, and genotype}

The severity of the phenotype, defined by a high DI score, appeared to be associated with an early age at onset of symptoms (0.3 months [IQR25-75: 0.01-5] for patients with DI score $>5$ vs 3 months [IQR25-75: 2-5] for patients with DI score $\leq 5(p=0.008))$, however, this was not significantly related to the type of symptoms at onset (plegic attacks, paroxysmal dystonic attacks or ocular movements).

The p.E815K ATP1A3 mutation was significantly associated with a more severe phenotype with a mean DI score of $12.3( \pm 2.5)$ vs. $7.4( \pm 2.1)$ for p.D801N and $6.7( \pm 3.6)$ for other mutations $(p=0.011)$. Patients who displayed an episode of regression tended to be more severely neurologically impaired at the last follow-up visit (DI score: $9.857 \pm 3.388$ vs. $7 \pm$ 3.302; $p=0.059$ ). Bradykinesia, recorded in 16 patients, was associated with an early age at assessment in this cohort $(p<0.01)$, and no other correlation could be established (in particular, for dystonia and/or chorea, age at onset, or mutation).

Dystonia (nonparoxysmal), observed in 16 patients, was correlated with a later age at assessment $(p=0.007)$. Patients with dystonia and/or chorea $(n=18)$ were compared to those without dystonia or chorea $(n=10)$. The former tended to be older at inclusion (14.0 years [IQR $25-75: 8.4-17.8]$ vs 4.4 years [2.8-19.7]; $p=0.115)$ with a significantly earlier age at onset (0.3 months [IQR $25-75: 0.1-2.5]$ vs 2.5 [2-5]; $p=0.042)$, more pronounced hypotonia $(77.8 \%$ vs $20 \% ; p=0.011$ ), and more severe neurological impairment (median DI score: 6.5 [IQR $25-75: 5-$ $8]$ vs 3 [3-6]; $p=0.012$ ). The latter was calculated using a modified DI score without dystoniachorea items. No association was found with a history of regression $(p=1.000)$, status epilepticus $(p=0.226)$, or severity of impairment of speech intelligibility $(p=0.24)$. The pattern of impairment (regarding walking ability, gross/fine motor function, communication, and behavioural or intellectual abilities, as assessed using the AHC disability index) was highly variable among children, and no specific pattern of neurological impairment associated with the presence of dystonia or chorea was apparent. In addition, no correlation was established between the occurrence of chorea or dystonia and genotype.

\section{Discussion}

Our study cohort reveals that patients with AHC harbouring ATP1A3 mutation exhibit various forms of hyperkinetic NPMDs (chorea, dystonia, myoclonus, and ataxia). Although dystonia or

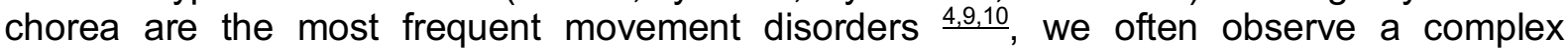
phenotype of mixed NPMDs with a heterogeneous degree of severity. However, our observations reveal two features that are characteristic of this cohort. First, all patients (of all ages) were found to be hypotonic, a finding frequently observed in AHC patients in other series 4,11. Second, dystonia or chorea correlated with a degree of hypotonia clinically perceived as more severe. Thus, in the same patient, an association between chorea-dystonia and marked hypotonia is often present; i.e. the patient is "soft" (hypotonic) and "stiff" (dystonic) at the same time, and this coexistence of both characteristics is striking. Patients can have a dystonic posture of one or more limbs or of axial muscles (i.e. torticollis), mixed with marked hypotonia on passive mobilisation either of the same limb or the opposite side limbs or can have dystonia of the extremities and central hypotonia at the same time. All possible variations of coexistence of dystonia and hypotonia have been seen, differing from one patient to another, but remaining specific for each individual patient.

The ATP1A3 gene encodes for the $\alpha 3$ subunit of the $\mathrm{Na}+\mathrm{K}+$ ATPase, which is neuron-specific and highly expressed in the cortex, as well as the basal ganglia $\underline{12}$. The cerebellum appears 
to be highly susceptible to dysfunction of the $\alpha 3$ subunit, possibly because Purkinje cells

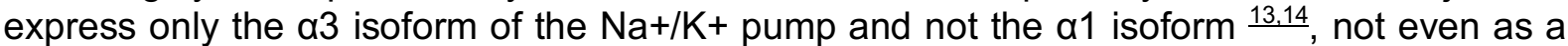
compensatory response to $\alpha 3$ isoform dysfunction $\frac{15}{-1}$. In patients' neurons, reduced affinity of the $\mathrm{Na}^{+} / \mathrm{K}^{+}$ATPase for $\mathrm{Na}^{+}$leads to elevated intracellular $\mathrm{Na}^{+} \underline{16}$. Since the neuronal uptake of dopamine relies on an appropriate $\mathrm{Na}^{+}$gradient, increased intracellular $\mathrm{Na}^{+}$may impair uptake and ultimately lead to dystonia $\frac{17}{-} \mathrm{Na}^{+} / \mathrm{Ca}^{2+}$ imbalance could also alter neurotransmitter uptake and abnormal neuronal firing; a relevant mechanism in the pathophysiology of dystonia $\underline{18,19}$. These pathophysiological mechanisms could explain why, in the same patient, we can observe hypotonia (implicating the cerebellum) and dystonia (dopamine-related) at the same time. Hypotonia (with or without dystonia) is a consistent feature that persists in adulthood, and should be added to the list of specific motor patterns associated with the disorder, but has yet to be specifically described.

In our study there seems to be a discrepancy with the results of prior studies in French patients

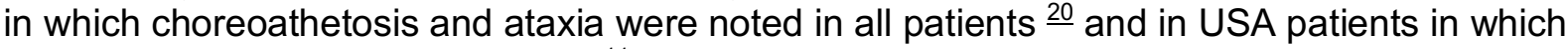
ataxia was noted in $68 \%$ of patients ${ }^{11}$. Phenomenological assessment of movement disorders may be very challenging in childhood; this was suggested by authors of the former study for AHC patients (Bourgeois et $\mathrm{al}^{20}$ ) ) and was further highlighted by a recent study that reported on young-onset movement disorders patients referred to a multidisciplinary outpatient clinic $\underline{21}$. The advantage of our study is its prospective nature with clinical examination particularly focalized in assessment of movement disorders, completed by a further blinded video-based analysis of phenomenology by our second movement disorder expert.

Although a phenotype-genotype correlation was confirmed in our population concerning the severity of the disease (the $\mathrm{pE} 815 \mathrm{~K}$ mutation correlated with severity) in agreement with

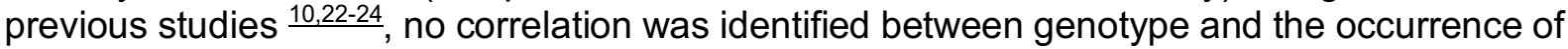
dystonia and/or chorea. Therefore, we are unable to confirm previously reported trends, which implied that different mutations were associated with different NPMD types $\frac{10}{}$, however, it should be noted that this latter study was based on retrospective investigation without a specific focus on movement disorders, in contrast to the present study.

According to our results, there is a significant interrelationship between the presence of dystonia or chorea and the severity of disability based on DI score, which accounts for global neurological impairment. This result was still significant even based on modified DI scores, excluding dystonia/chorea items. Clearly, these two NPMDs represent an additional handicap in everyday-life activities and when present, result in a poorer quality of life and a higher disability score. Dystonia and chorea therefore appear to be predictors or hallmarks of further disability, as well as contributing to the overall clinical presentation. Moreover, an earlier age at onset seems to be a poor predictive factor for an overall more severe disabling phenotype, as well as for later appearance of dystonia and/or chorea. Motor regression occurred in $25 \%$ of this series of patients, none of them regained prior level of function; data collection was insufficient to determine if they exhibited appearance of new or exacerbation of previously existing movement disorder (as is the case in patients with rapid onset dystonia parkinsonism).

The presence of bradykinesia in patients of this cohort was related to an earlier age at assessment. This gives rise to the hypothesis that bradykinesia is a constituent of the natural motor evolution of the disease, becoming less prominent with age. On the contrary, dystonia or chorea tended to be present in older patients. It is noteworthy that Masoud et al $\stackrel{5}{5}$, in their study of motor function domains in AHC, did not observe a worse motor function in older patients, although movement disorders were not specifically studied $\underline{5}$. These observations should be investigated in larger longitudinal cohort studies. In our study, significant dysarthria or impaired speech intelligibility were frequent, and contribute to the functional disturbance in AHC patients. This is consistent with the study of Masoud et al $\stackrel{5}{ }$, in which speech was shown to be the most affected function.

Regarding treatment practices, it is worth mentioning that only one of the patients included in our study was receiving a medication specifically targeting a movement disorder. Although we 
did not collect data on previously used treatments, this is a rather surprising finding, particularly considering that dystonia and/or chorea interfere with everyday motor function and significantly contribute to overall disability in patients with $\mathrm{AHC}$. Current therapies for $\mathrm{AHC}$ are usually assessed according to their efficacy on paroxysmal movement disorders (plegic or paroxysmal dystonic attacks), which undoubtedly represent devastating features of the disease, whereas the effects on NPMDs are poorly documented $\frac{25}{2}$. We should probably also take into account the fact that therapeutic resources are not considered efficacious for rapid-onset dystonia parkinsonism- an allelic disorder related to ATP1A3 in which the dystonic component predominates. Standard dopaminergic therapy, including L-dopa, is ineffective and most of the drugs available to treat dystonia often do not show any benefit 3 .

We suggest that a prospective study on progression of NPMDs in AHC is needed, to better define their impact on the severity of the disease and quality of life. The next step would then be the design of a controlled therapeutic trial, focusing on drugs for the treatment of the nonparoxysmal movement disorder components in $\mathrm{AHC}$, including evaluation of optimal doses.

A limitation of this study is the cross-sectional design with little or no information on the exact age of appearance of the various NPMDs and their evolution with time. Furthermore, statistical analyses were exploratory due to the numerous tests done, the relatively limited number of patients, and to the cross-sectional nature of the study. However our study provides new information on one of the most debilitating features of AHC, which is non-paroxysmal movement disorders. Our findings also contribute to further define the clinical phenotypes in $\mathrm{AHC}$ and pinpoint the need for treatment trials, with impact on NPMDs as a primary endpoint. 


\section{Figure legends}

Figure 1. Clinical characteristics of the patients

Results of nonparoxysmal disability index (DI) (column 1-7), and speech intelligibility scale among our patients. For movement disorders, score of 0 means absence of movement disorders.

\section{Figure 2. Movement disorders among patients}

Repartition of the various types of abnormal movements among the 28 patients. 


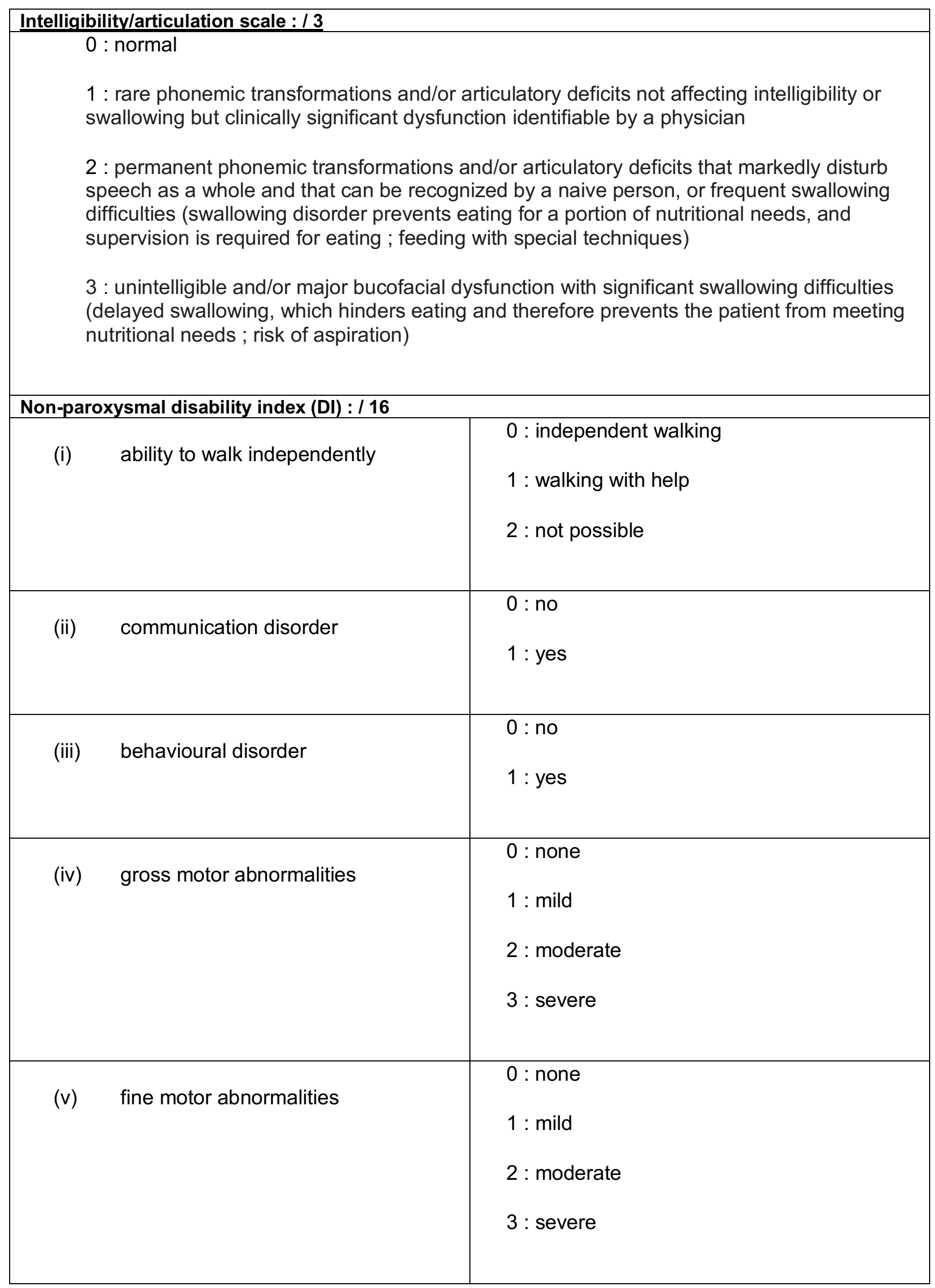




\begin{tabular}{|ll|l|}
\hline (vi) $\begin{array}{l}\text { movement disorders (chorea, } \\
\text { dystonia, myoclonus, tremor and } \\
\text { complex movement disorders) }\end{array}$ & $1:$ mild \\
& $2:$ moderate \\
& $3:$ severe \\
\hline (vii) Intellectual disability & $0:$ none \\
& & $1:$ mild \\
& $2:$ moderate \\
& $3:$ severe \\
\hline
\end{tabular}

Table 1. Intelligibility Scale and Non-paroxysmal Disability Index used to assess the patients 


\begin{tabular}{|c|c|c|c|}
\hline Patients & & $\mathbf{N}$ & \\
\hline \multirow{3}{*}{ Sex } & & 28 & \\
\hline & Males & & $15(53.6 \%)$ \\
\hline & Females & & $13(46.4 \%)$ \\
\hline $\begin{array}{l}\text { Age at inclusion } \\
\text { (years) }\end{array}$ & & & $\begin{array}{c}11 \\
(4.4-18 .) \\
{[1.8-38.7]}\end{array}$ \\
\hline \multirow[t]{5}{*}{ Genetics } & & 28 & \\
\hline & p.D801N mutation & & $9(32.1 \%)$ \\
\hline & p.E815K mutation & & $4(14.3 \%)$ \\
\hline & p.G947R mutation & & $4(14.3 \%)$ \\
\hline & Other pathogenic mutation & & $11(39.3 \%)$ \\
\hline \multicolumn{4}{|c|}{ 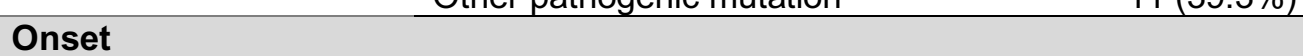 } \\
\hline Age at onset (months) & & & $\begin{array}{c}2 \\
(0.06-3) \\
{[0.03-3]}\end{array}$ \\
\hline \multirow[t]{5}{*}{ Mode of onset } & & 28 & \\
\hline & Plegic attacks & & $5(17.9 \%)$ \\
\hline & Paroxysmal dystonic attacks & & $9(32.1 \%)$ \\
\hline & Abnormal eye movements & & $6(21.4 \%)$ \\
\hline & $\begin{array}{l}\text { Other (hypotonia, seizures, } \\
\text { autonomic dysfunction) }\end{array}$ & & $8(28.6 \%)$ \\
\hline \multicolumn{4}{|l|}{ Medical follow-up } \\
\hline \multirow[t]{3}{*}{ Plegic attacks } & Occurrence of plegic attacks & 28 & $28(100 \%)$ \\
\hline & Age at onset (months) & 27 & $\begin{array}{c}7 \\
(2-10) \\
{[0.06-24]}\end{array}$ \\
\hline & Recurrence until inclusion & 27 & $20(74.1 \%)$ \\
\hline \multirow[t]{3}{*}{$\begin{array}{l}\text { Paroxysmal dystonic } \\
\text { attacks }\end{array}$} & $\begin{array}{l}\text { Occurrence of paroxysmal dystonic } \\
\text { attacks }\end{array}$ & 28 & $25(89.3 \%)$ \\
\hline & Age at onset (months) & 20 & $\begin{array}{c}3 \\
(1.5-3) \\
{[0.03-96]}\end{array}$ \\
\hline & Recurrence until inclusion & 25 & $20(80 \%)$ \\
\hline \multirow[t]{2}{*}{$\begin{array}{l}\text { Abnormal eye } \\
\text { movements }\end{array}$} & $\begin{array}{l}\text { Occurrence of abnormal eye } \\
\text { movements }\end{array}$ & 27 & $25(92.6 \%)$ \\
\hline & Age at onset (months) & & $\begin{array}{c}3.5 \\
(2-7) \\
{[0.03-84]} \\
\end{array}$ \\
\hline \multirow[t]{3}{*}{ Epileptic seizures } & Occurrence of epileptic seizures & 28 & $11(39.3 \%)$ \\
\hline & Median age at onset ${ }^{*}$ & 11 & $\begin{array}{c}1.5 \\
(0.5-5.0) \\
{[0-11]}\end{array}$ \\
\hline & Status epilepticus & 11 & $6(54.6 \%)$ \\
\hline \multirow{3}{*}{$\begin{array}{l}\text { Psychomotor } \\
\text { regression }\end{array}$} & Occurrence of regression & 28 & $7(25.0 \%)$ \\
\hline & Age at onset (months) & 7 & $\begin{array}{c}10 \\
(1.6-17) \\
{[0.6-19]}\end{array}$ \\
\hline & $\begin{array}{l}\text { More than one episode of } \\
\text { regression }\end{array}$ & 0 & $0(0 \%)$ \\
\hline \multirow[t]{5}{*}{ Intellectual disability } & & 28 & \\
\hline & no & & 3 \\
\hline & mild & & 9 \\
\hline & moderate & & 13 \\
\hline & severe & & 3 \\
\hline \multirow{4}{*}{$\begin{array}{l}\text { Hyperkinetic } \\
\text { movement disorders at } \\
\text { baseline * }\end{array}$} & Dystonia & 28 & $16(57.1 \%)$ \\
\hline & Chorea & & $10(35.7 \%)$ \\
\hline & Myoclonus & & $4(14.3 \%)$ \\
\hline & Cerebellar ataxia & & $2(7 \%)$ \\
\hline
\end{tabular}




\begin{tabular}{llcc}
\hline & Tremor & $0(0 \%)$ \\
\cline { 2 - 3 } & No movement disorder & $8\left(28.5 \%^{\circ}\right.$ \\
\hline \multirow{2}{*}{ Others } & Pyramidal tract signs & 27 & $5(18.5 \%)$ \\
\cline { 2 - 3 } & Bradykinesia & 28 & $16(57.1 \%)$ \\
\cline { 2 - 3 } & Rostrocaudal gradient & 21 & $15(71.4 \%)$ \\
\hline
\end{tabular}

Table 2. Clinical and genetic characteristics of the $28 \mathrm{AHC}$ patients. *Various types of hyperkinetic movement disorders may be mixed in the same patient.

Quantitative variables were presented as median (IQR25-75) [min-max] and categorical variables with $\mathrm{n}(\%) . \mathrm{N}$ : Number of patients with available data. 
Appendix 1

Name Location

Eleni Panagiotakaki University Hospitals of Lyon, France

Diane Doummar

Trousseau

Hospital,Paris,

France

Erika Nogue

University Hospital, Montpellier, France
Role

Author

Author

Author
Contribution

Major role in the

acquisition of data ;

drafted the

manuscript for

intellectual content

Major role in the

acquisition of data ;

revised the

manuscript for

intellectual content

Major role in the

acquisition of the

biostatistical data ;

interpretation of the

data 
Nicolas Nagot

Gaetan Lesca

Florence Riant

Sophie Nicole

Alexis

Arzimanoglou

Agathe Roubertie

University Hospital, Montpellier, France

Institut de génétique

Fonctionnelle,

Montpellier

University, France

University Hospitals Author

of Lyon, France

Author

Charlene Delaygue

University Hospital,

Montpellier, France

Marie Anne Barthez University Hospital,

Tours, France

Marie Cécile

Nassogne

Anne Dusser

Louis Vallée

Thierry Billette

Marie Bourgeois

Christine loos

Cyril Gitiaux

Cécile Laroche

Mathieu Milh
Cliniques

Universitaires Saint-

Luc, Brussels,

Belgium

Bicêtre Hospital,

Paris, France

University Hospital,

Lille, France

Trousseau

Hospital,Paris,

France

Necker Hospital, Author

Paris, France

Diderot University, Author

Paris, France

Necker Hospital, Author

University Hospital, Author

Limoges, France

University Hospital, Marseille, France

Author

Author

Author
Paris, France
Author

Author

Clinical data acquisition

Clinical data acquisition

Clinical data acquisition

Clinical data acquisition

Clinical data acquisition

Clinical data acquisition

Clinical data acquisition

Clinical data acquisition 
Vincent Des Portes University Hospitals Author

Clinical data acquisition of Lyon, France 


\section{References}

1. Sweney MT, Silver K, Gerard-Blanluet M, et al. Alternating Hemiplegia of Childhood: Early Characteristics and Evolution of a Neurodevelopmental Syndrome.

PEDIATRICS. 2009 Mar 2;123(3):e534-e541.

2. Heinzen EL, Arzimanoglou A, Brashear A, Clapcote SJ, Gurrieri F, Goldstein DB, et al. Distinct neurological disorders with ATP1A3 mutations. Lancet Neurol. 2014 May;13(5):503-14.

3. Carecchio M, Zorzi G, Ragona F, Zibordi F, Nardocci N. ATP1A3-related disorders: An update. Elsevier Ltd; 2018 Feb 10;:1-7.

4. Panagiotakaki E, Gobbi G, Neville B, et al. Evidence of a non-progressive course of alternating hemiplegia of childhood: study of a large cohort of children and adults. Brain. 2010 Oct 24;133(12):3598-3610.

5. Masoud M, Gordon K, Hall A, et al. Motor function domains in alternating hemiplegia of childhood. Dev Med Child Neurol. 2017 Aug;59(8):822-828.

6. Jasien JM, Bonner M, D'alli R, et al. Cognitive, adaptive, and behavioral profiles and management of alternating hemiplegia of childhood. Dev Med Child Neurol. 2019 May;61(5):547-554.

7. Aicardi J. Alternating Hemiplegia of Childhood. New York, Raven Press 1994

8. Fahn S. Classification of movement disorders. Mov Disord. 2011 May 27;26(6):947957.

9. Ishii A, Saito Y, Mitsui J, et al. Identification of ATP1A3 mutations by exome sequencing as the cause of alternating hemiplegia of childhood in Japanese patients. Speletas M, editor. PLoS ONE. 2013;8(2):e56120.

10. Panagiotakaki E, De Grandis E, Stagnaro M, et al. Clinical profile of patients with ATP1A3 mutations in Alternating Hemiplegia of Childhood-a study of 155 patients. Orphanet J Rare Dis. Orphanet Journal of Rare Diseases; 2015 Sep 23;:1-13.

11. Mikati MA, Kramer U, Zupanc ML, Shanahan RJ. Alternating hemiplegia of childhood: clinical manifestations and long-term outcome. Pediatr Neurol. 2000 Aug;23(2):134141.

12. McGrail KM, Phillips JM, Sweadner KJ. Immunofluorescent localization of three Na,KATPase isozymes in the rat central nervous system: both neurons and glia can express more than one Na,K-ATPase. Journal of Neuroscience. 1991 Feb;11(2):381391.

13. Ikeda K, Satake S, Onaka T, et al. Enhanced inhibitory neurotransmission in the cerebellar cortex of Atp1a3-deficient heterozygous mice. J Physiol (Lond). 2013 Jul 1;591(13):3433-3449.

14. Peng L, Martin-Vasallo P, Sweadner KJ. Isoforms of Na,K-ATPase alpha and beta subunits in the rat cerebellum and in granule cell cultures. Journal of Neuroscience. 1997 May 15;17(10):3488-3502.

15. Isaksen TJ, Kros L, Vedovato N, et al. Hypothermia-induced dystonia and abnormal cerebellar activity in a mouse model with a single disease-mutation in the sodium- 
potassium pump. Petrou S, editor. PLoS Genet. 2017 May;13(5):e1006763.

16. Rodacker V, Toustrup-Jensen M, Vilsen B. Mutations Phe785Leu and Thr618Met in $\mathrm{Na}+, \mathrm{K}+-\mathrm{ATPase}$, associated with familial rapid-onset dystonia parkinsonism, interfere with $\mathrm{Na}+$ interaction by distinct mechanisms. J Biol Chem. 2006 Jul 7;281(27):1853918548.

17. Blanco-Arias $P$, Einholm AP, Mamsa $H$, et al. A C-terminal mutation of ATP1A3 underscores the crucial role of sodium affinity in the pathophysiology of rapid-onset dystonia-parkinsonism. Hum Mol Genet. 2009 Jul 1;18(13):2370-2377.

18. Starr PA, Rau GM, Davis V, et al. Spontaneous pallidal neuronal activity in human dystonia: comparison with Parkinson's disease and normal macaque. J Neurophysiol. 2005 Jun;93(6):3165-3176.

19. Tang JKH, Moro E, Mahant N, et al. Neuronal firing rates and patterns in the globus pallidus internus of patients with cervical dystonia differ from those with Parkinson's disease. J Neurophysiol. 2007 Aug;98(2):720-729.

20. Bourgeois M, Aicardi J, Goutières F. Alternating hemiplegia of childhood. J Pediatr. 1993 May;122(5 Pt 1):673-679.

21. van Egmond ME, Eggink $\mathrm{H}$, Kuiper A, et al. Crossing barriers: a multidisciplinary approach to children and adults with young-onset movement disorders. J Clin Mov Disord. 2nd ed. 2018;5(1):3.

22. Sasaki M, Ishii A, Saito $Y$, et al. Genotype-phenotype correlations in alternating hemiplegia of childhood. Neurology. 2014 Feb 11;82(6):482-490.

23. Yang X, Gao H, Zhang J, et al. ATP1A3 mutations and genotype-phenotype correlation of alternating hemiplegia of childhood in Chinese patients. Zhou F, editor. PLoS ONE. 2014;9(5):e97274.

24. Viollet L, Glusman G, Murphy KJ, et al. Alternating Hemiplegia of Childhood:

Retrospective Genetic Study and Genotype-Phenotype Correlations in 187 Subjects from the US AHCF Registry. Zhou F, editor. PLoS ONE. 2015 May 21;10(5):e0127045-14.

25. Haffejee S, Santosh PJ. Treatment of alternating hemiplegia of childhood with aripiprazole. Dev Med Child Neurol. 2009 Jan;51(1):74-77. 


\section{Acknowledgements}

We acknowledge patients and their families for participating in the study, and the French AHC association for support; Oliver Gubbay for reviewing English; Doctor Pierre Meyer for editing videos, and Dr Lagavulin for discussions.

\section{Funding:}

None

\section{Conflict of interest}

The authors report no disclosures relevant to the manuscript.

\section{Video legend}

Video 1. 15-years-old female patient. Part I: when walking: dystonic posture of the left foot, with reduced balance of the left upper limb and increased swinging of the right arm . Part II: passive tonus assessment, showing baseline decreased muscle tone in the right arm and mild increased muscle tone in the left arm. Part III: bradykinetic and dystonic movements when the patient is asked to oppose her fingers to thumb; note facial hypomimia.

This female patient is the only child of healthy non-consanguineous parents. She was born after an uneventful pregnancy by vaginal delivery. First paroxysmal events started at the age of 2 months (abnormal ocular movements - unilateral nystagmus). First hemiplegic attacks started at the age of 9 months with disappearance of symptoms immediately following sleep. Diagnosis was made by Pr Jean Aicardi.

At last follow-up this 15-years-old patient had a borderline intellectual functioning and attended normal school, but with many special arrangements. She still experienced hemiplegic events characterized by an inability to speak and to swallow and (painful) hemi-dystonic attacks involving the mouth and the tongue. Paroxysmal episodes were triggered by stress or excitement, bathing, cold temperature, sunlight. She never experienced epileptic seizures. Permanent motor dysfunction was more obvious on the left side of the body (see video).

She was treated by clonazepam, flunarizine, clobazam, acetazolamide. Flunarizine treatment was the most efficient.

Genetic testing identified the c.2411C>T (p.T804I) de novo mutation in ATP1A3 gene.

Video 2. 4.5-years-old male patient. Axial hypotonia with kyphotic sitting; Permanent choreic movements involving the face, the upper limbs, associated to dystonic posture of the feet.

This male patient is the second child of healthy unrelated parents. He was born by cesarean section with uneventful perinatal period. The patient was referred at 2.5 months for hypotonia and unilateral nystagmus noticed form the first weeks of life. Paroxysmal dystonic attacks involving one body side occurred at 3 months of age, and recurred during the next 3 months. From the age of 6 months, the patient exhibited episodes of quadriplegic attacks sometimes followed by epileptic seizures, triggered by fever or without obvious triggering factor; quadriplegic attacks progressively evolved to hemiplegic attacks that still occurred 3 times a month at last follow-up. This patient aged 4.5 years at last follow-up was able to sit with axial hypotonia, he emitted sounds. Treatment included flunarizine (with poor benefit on plegic attacks), sodium valporate and lamotrigine. Genetic testing disclosed de novo c.2443G>A (p.Glu815 lys) mutation, in ATP1A3 gene.

Video 3. 35-years-old man. Part I: upper limb dystonia with right-sided predominance, and writer's cramp. Part II: Mouth dystonia when talking with dystonic dysarthria; note the torticollis and facial hypomimia. Part III: when walking: trunk and lower limb dystonia; note the 
bradykinesia. Part IV: passive tonus assessment, showing baseline decreased muscle tone on both upper limbs.

This male patient is the offspring of healthy non-consanguineous parents. First paroxysmal events started at the age of 2 months and were hemiplegic attacks with disappearance of symptoms immediately following sleep. Later he began having paroxysmal dystonic attacks and abnormal ocular movements. He has never experienced epileptic seizures. Diagnosis was made at the age of 2 years.

He had a normal intellectual functioning and attended normal school, but with many special arrangements. He spoke early at the age of 15 months with phrases, but he is now very dysarthric, with a deterioration of his dysarthria at the adult age. He still experiences some hemiplegic events, but mostly paroxysmal dystonic attacks, with no identified trigger factors. He no longer experiences abnormal ocular movements. Until the age of 12 he could walk, but after his 13th birthday he had a deterioration after a head trauma. He can no longer walk in autonomous manner (he is able to walk at home with help) and presents marked dystonia. He studied graphic design and web site conception but has never been able to find an employment.

He was treated in the past by flunarizine, that he stopped taking before adulthood because it was no effective. He is not receiving any drugs and refuses introduction of any treatment.

On 2013 a genetic testing identified the c.791_866delinsTTCTGGG mutation in ATP1A3 gene, that was not found in mother's DNA, but father was not available for testing. 


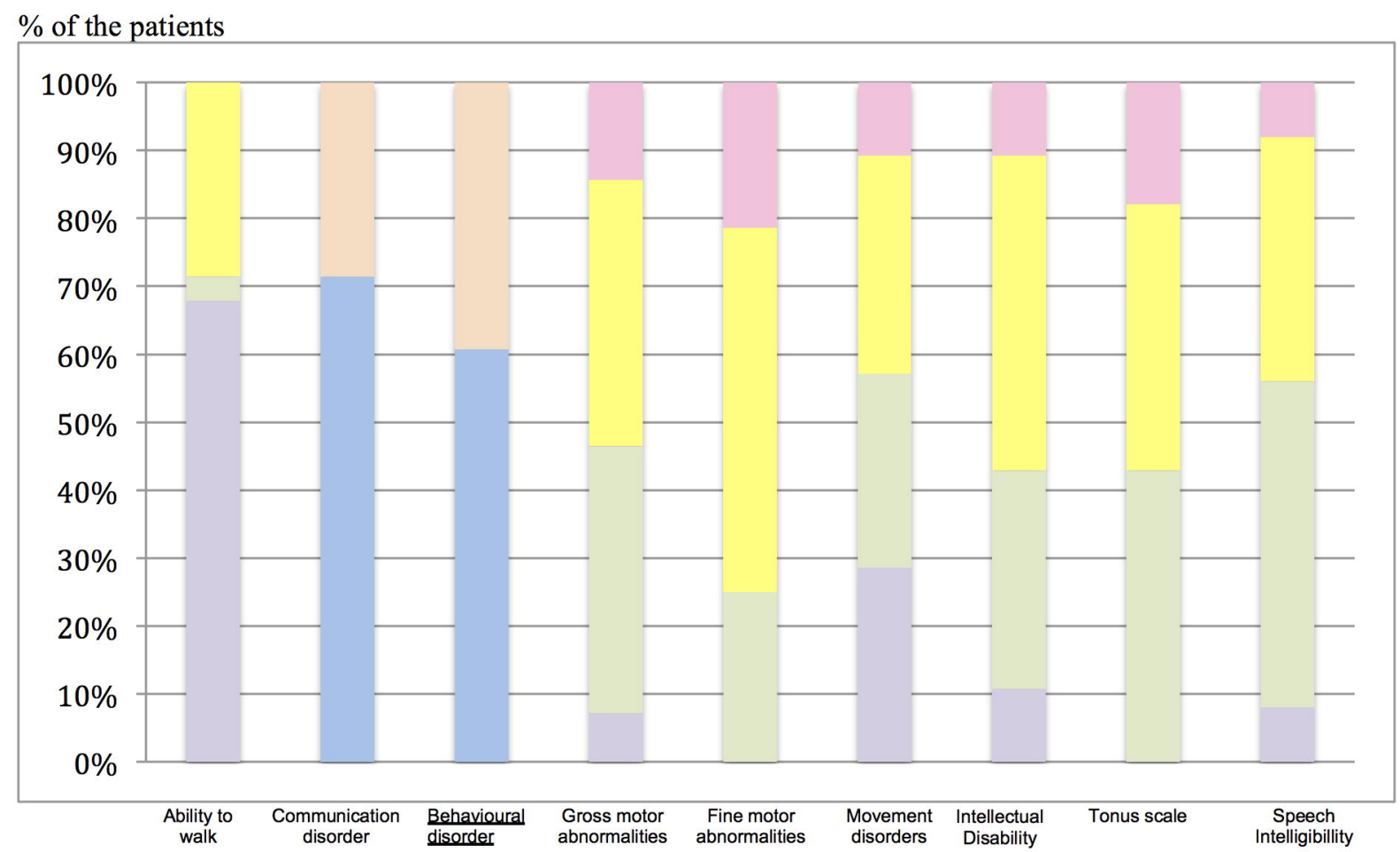

\begin{tabular}{|ll|}
$\square$ & score $=0$ \\
$\square$ & score=1 \\
$\square$ & score=2 \\
$\square$ & score=3 \\
$\square$ & no \\
$\square$ & yes \\
\hline
\end{tabular}

Figure 1. 


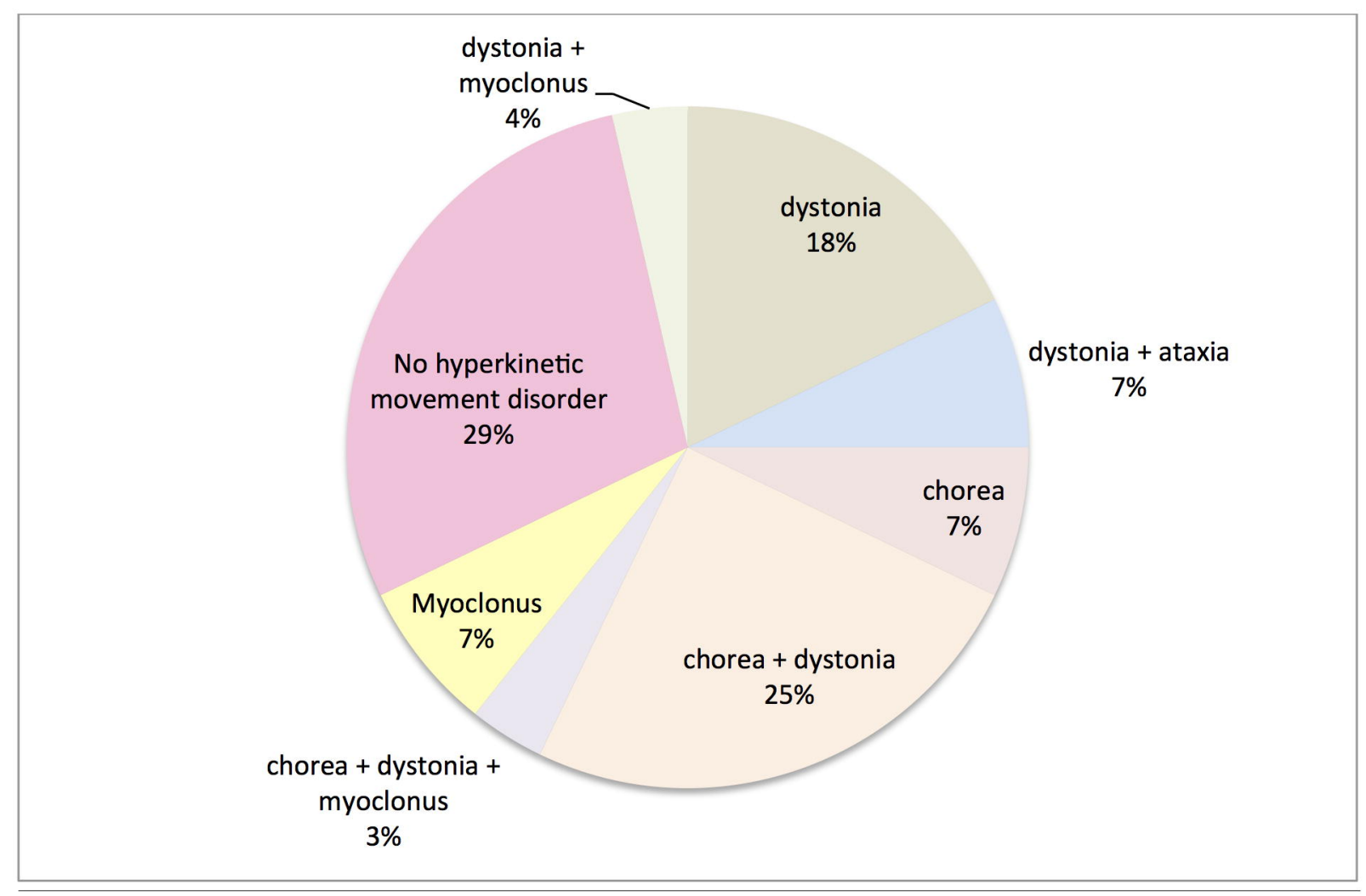

Figure 2. 